\title{
Antimicrobial resistance of bacterial isolates from urinary tract infections at Felge Hiwot Referral Hospital, Ethiopia
}

\author{
Fantahun Biadglegne ${ }^{1}$, Bayeh Abera ${ }^{1}$
}

\begin{abstract}
Urinary tract infections are common health problems and vary according to geography and regions. A retrospective analysis was conducted to determine the antimicrobial resistance of bacterial isolates from urine at Felege Hiwot Referral Hospital from September 2003 to June 2008. From 529 urine specimens, bacterial isolates were found in 160 [(30.2\%) (95\% CI: $26.3-34.1 \%)]$. Of these, $116(72.5 \%)$ of the isolates were gram negatives. Single and multiple drug resistance to the commonly used antibiotics were high among bacterial isolates in the area. Thus, rational use of drugs should be practiced. [Ethiop. J. Health Dev. 2009;23(3):236-238]
\end{abstract}

\begin{abstract}
Introduction
Urinary tract infections (UTIs) caused by bacteria associated with an increased antimicrobial resistant species are common in all age groups (1). Most urinary tract infections are initiated by organisms that gain entrance from the natural environment to the bladder through the urethra and are more common in women than men (2).Urinary pathogens vary depending upon age, sex, catheterization, hospitalization and previous exposure of antimicrobials (3).The prevalence of antimicrobial resistance with urinary tract infection is increasing and varies according to geographical and regional location (4), therefore up to date information is essential for clinicians for appropriate antimicrobial therapy. To our knowledge, no microbiological data exist from urinary tract infections in Bahir Dar. The purpose of this survey was to determine the antibiotic susceptibility patterns of bacterial isolates from patients with urinary tract infections at Felege Hiwot Referral Hospital, Bahir Dar, Ethiopia.
\end{abstract}

\section{Methods}

This cross-sectional study was conducted based on retrospective review of records of 529 patients for whom urine specimens were processed for culture and susceptibility tests at Bahir Dar Regional Health Research Laboratory from September 2003 to June 2008. Standard operating procedures of Feingold and Martin (5) were followed to isolate and identify the bacteria and data were collected from log book using a format. Urine samples were inoculated on cystine lysine electrolyte deficient (CLED) agar and blood agar plate (Oxoid Basingstoke, UK) using standard $4 \mathrm{~mm}$ diameter wire loop and incubated at $37^{\circ} \mathrm{C}$ for 24 hours aerobically.

Records showed that antimicrobial susceptibility test was done on Muller-Hinton agar (Oxoid Basingstoke,UK) with disc diffusion technique of Bauer et al (6) against ampicillin $(10 \mu \mathrm{g})$, gentamycin $(10 \mu \mathrm{g})$, penicillin $(10 \mathrm{IU})$, tetracycline $(30 \mu \mathrm{g})$, chloramphinicol $(30 \mu \mathrm{g})$, erythromycin $(30 \mu \mathrm{g})$, nitrofurantoin $(300 \mu \mathrm{g})$, cotrimoxazole $(25 \mu \mathrm{g})$, streptomycin $(10 \mu \mathrm{g})$, carbenicillin
$(100 \mu \mathrm{g})$, cephalotin $(30 \mu \mathrm{g})$ and norfloxacin $(10 \mu \mathrm{g})$. Reference strains, E.coli ATCC 25922 and S.aureus ATCC 25923, were used as controls. Ethical clearance was obtained from the ethical committee of Medicine and Health Sciences, Bahir Dar University.

\section{Results}

Urine samples from 529 patients were analyzed in the study. Female patients constituted 308 (58.2\%). The age range was from 2 months to 89 years. Three hundred sixty eight $(69.6 \%)$ were from out patients and 161 (30.4\%) were inpatients. Bacterial isolates were found in 160 [(30.2\%) (95\% CI: 26.3-34.1\%)] urine specimens, of these $107(67 \%)$ were from females. One hundred sixteen $(72.5 \%)$ of the isolates were gram negative bacteria. E.Coli was the predominant isolate $77(48 \%)$ followed by S.aureus 31(19.4\%) (Table 1).

Bacterial isolates from patients with UTI showed high levels of single and multiple antimicrobial resistance against commonly prescribed drugs as shown in Table1. The overall multiple drug resistance was $93.1 \%$, while $2.5 \%$ of the isolates were resistant to one antibiotic and $4.4 \%$ were sensitive to all antibiotics tested.

\section{Discussion}

In the present study, the isolation rate of bacteria was $30.2 \%$. This is in accordance with previous reports from Gondar (7) and Addis Ababa (8). However, it is lower than other previous reports from Gondar of $39.5 \%$ (3) and $36.6 \%$ (9). We found that $72.5 \%$ of the etiologic agents of UTIs were gram negative enteric bacteria, $E$.coli being the most predominant, which is similarly reported elsewhere in the country (7). The prevalence of UTI was higher in female than male patients. This might be due to the fact that shorter, wider urethra and its proximity to the anus is the predisposing factor for infection. The isolation rate of S.aureus (19.4\%) is higher compared to the reports of Gondar which was $9.7 \%$ (9), $14.5 \%$ (7), $18 \%$ (3).

${ }^{T}$ Department of Medical Microbiology, Immunology and Parasitology, College of Medicine and Health Science, University of Bahir Dar, P.O. Box 1498, E-mail: fanta17@yahoo.com, Tel. 0911534989, Bahir Dar, Ethiopia 